Pacific Journal of Mathematics

POSITIVE DEFINITE FUNCTIONS WHICH VANISH AT 


\title{
POSITIVE DEFINITE FUNCTIONS WHICH VANISH AT INFINITY
}

\author{
Alessandro Figà-Talamanca
}

Let $G$ be a separable noncompact locally compact group. Let $A(G)$ and $B(G)$, respectively, be the Fourier algebra and the Fourier-Stieltjes algebra of $G$ as defined by P. Eymard. We prove that if $G$ is unimodular and satisfies an additional hypothesis, which implies noncompactness, there exists an element of $B(G)$, indeed a positive definite function, which vanishes at infinity but is not in $A(G)$. This function actually belongs to $B^{\rho}(G)$, that is, it defines a unitary representation of $G$ which is weakly contained in the regular representation.

We refer to [3] for the definitions and properties of $A(G), B(G)$, $B_{\rho}(G)$ and of the related spaces $V N(G), C^{*}(G)$ and $C_{\rho}^{*}(G)$.

We recall only that $C_{\rho}^{*}(G)$ and $V N(G)$ are, respectively, the $C^{*}$ algebra and the von Neumann algebra, generated by $L^{1}(G)$ acting by left convolution on $L^{2}(G)$. While $C^{*}(G)$ is the $C^{*}$-algebra of the group obtained by completing the algebra $L^{1}(G)$ with respect to the norm $\|f\| C^{*}(G)=\sup _{\pi \in \Sigma}\|\pi(f)\|$, where $\Sigma$ is the space of all $*$-representations of $L^{1}(G)$ as an algebra of operators on a Hilbert space. We also recall that $B(G)$ is, in a natural fashion, the dual of $C^{*}(G)$, while $B_{\rho}(G)$ is the dual of $C_{\rho}^{*}(G)$ which is a quotient algebra of $C^{*}(G)$. Finally $V N(G)$ is the dual of $A(G)$;

When $G$ is commutative and $\hat{G}$ is the character group of $G$, $A(G)$ and $B(G)$, respectively, coincide with the algebra of Fourier transforms of elements of $L^{1}(\widehat{G})$ and the algebra of Fourier-Stieltjes transforms of bounded regular measures on $\hat{G}$.

Thus for $G$ commutative our result reduces to the classical theorem which asserts that on any nondiscrete locally compact abelian group $\hat{G}$, one can construct a singular measure with Fourier-Stieltjes transform vanishing at infinity. This classical result was proved for the first time, for the case $\widehat{G}=T$ and $G=Z$, by M. D. Menchoff [10].

When $G$ is noncommutative the situation may be quite different: I. Khalil proved in [6] that if $G$ is the affine group of the line, i.e., the group of transformations $x \rightarrow a x+b$ of $\boldsymbol{R}$ into $\boldsymbol{R}$, then $B(G) \cap$ $C_{0}(G)=A(G)$.

Therefore some other hypothesis, in addition to noncompactness of $G$, is needed for our result to be true. In this paper we show that $A(G) \neq B(G) \cap C_{0}(G)$ provided that $G$ is unimodular and in addition satisfies the following condition: 
(H) The von Neumann algebra $V N(G)$ is not purely atomic.

This same hypothesis, which implies noncompactness, was used in [2] for another construction. A list of groups satisfying $(\mathrm{H})$ is given in [2]. An example of a noncompact unimodular group which does not satisfy $(\mathrm{H})$ is given in [1] and [7]. This example, as shown in [9] and in [13] does not satisfy the conclusion of the theorem. It seems possible that, for unimodular groups, $(\mathrm{H})$ is necessary as well as sufficient for the existence of functions of $B_{\rho}$ which vanish at infinity and are not in $A$. We also do not know whether, for nonunimodular groups condition $(\mathrm{H})$ is sufficient and/or necessary to yield the conclusion of the theorem.

The techniques used in this paper are related to those developed in [2] which in turn are related to the techniques of [4].

2. Preliminaries. The main device in proof of the theorem consists in transferring certain techniques of the theory of Fourier series on commutative compact groups to the present context. The simplest commutative infinite compact group is the Cantor group $D$ which we define as the product $D=\prod_{j=1}^{\infty}\{-1,1\}_{j}$, of countably many multiplicative groups of two elements. The character group $\hat{D}$ of $D$ can be simply described: first we have the Rademacher functions $r_{j}(t)=\varepsilon_{j}$ for $t=\left\{\varepsilon_{j}\right\} \in D$, which are simply the projections of an element into its $j$-th coordinate. The functions $r_{j}$ are of course characters of $D$ and also independent random variables with zero mean. All characters $w$ of $D$ may be obtained then as finite products of Rademacher functions. The characters of $D$ are called the Walsh functions and are numbered (according to R.E.A.C. Paley) as follows: $w_{0}=1, w_{n}=r_{j_{1}} \cdots r_{j_{s}}$ if $n=2^{j_{1}-1}+\cdots+2^{j_{s}-1}$, with $j_{1}<j_{2}<\cdots<j_{s}$. We shall indicate by $w$ a generic element of the character group $\hat{D}$ of $D$. We shall summarize in the following lemma a few well known technical results concerning Fourier series on $D$.

Lemma. Let $-1 \leqq \beta_{j} \leqq 1$ be a sequence of real numbers. Let $\dot{\phi}_{n}(t)=\prod_{j=1}^{n}\left(1+\beta_{j} r_{j}(t)\right)$, for $t \in D$. Then there exists a probability measure $\mu$ defined on the Borel subsets of $D$, such that

(1) $\phi_{n}(t)=\sum_{k=0}^{2 n} \widehat{\mu}\left(w_{k}\right) w_{k}(t)$

(2) $\hat{\mu}\left(w_{n}\right)=\beta_{j_{1}} \cdots \beta_{j_{s}}$ if $n=2^{j_{1}-1}+\cdots+2^{j_{s}-1}$, so that $\lim _{n} \hat{\mu}\left(w_{n}\right)=0$ if and only if $\lim _{j} \beta_{j}=0$

(3) $\mu$ is absolutely continuous if and only if $\Sigma \beta_{j}^{2}<\infty$. Thus if $\Sigma \beta_{j}^{2}=\infty$, no subsequence of $\Phi_{n}$ can converge in the norm of $L^{1}(D)$.

Proof. The measure $\mu$ is obtained as a weak* limit of the positive functions $\phi_{n}$ which have all integral one. Statements (1) and 
(2) are verified immediately. For (3) one proves that if $\Sigma \beta_{j}^{2}<\infty$, $\phi_{n} \in L^{2}(D)$, with bounded norms, and that if $\Sigma \beta_{j}^{2}=+\infty, \phi_{n}(t)$ converges to zero almost everywhere. For a more detailed proof cfr. [5, Th. 3.2. and Th. 4.1.].

We shall describe now the machinery which will allow us to bring to bear upon our problem ideas, results and techniques pertaining to Fourier analysis on the Cantor group. An analogous machinery was used in [2]. First of all, since we assume that $G$ is unimodular, we can make use, as in [2], of the canonical gace space $\Gamma=\left(L^{2}(G), V N(G), \operatorname{tr}\right)$, defined for a unimodular group $G$, by I. E. Segal [11], and extensively studied by R. A. Kunze [8] and W. F. Stinespring [12]. We refer to the cited papers and to [2] for the statements of the results needed here. We recall only that $A(G)$ is the image under an "inverse Fourier transform" of the space $L^{1}(\Gamma)$ of the operators which are measurable with respect to $V N$ and "absolutely integrable" with respect to the trace tr. The trace is defined as $\operatorname{tr}(P)=\|f\|_{L^{2}(G)}^{2}$, when $P$ is a projection and $P=L_{f}$ (left convolution by $f$ ), with $f \in L^{2}(G)$, and $\operatorname{tr}(P)=\infty$, otherwise. The fact that $G$ satisfies property $(H)$, which will be always assumed from now on, implies that there exists a projection $P$, of finite positive trace, which contains no minimal projections of $V N$. By choosing an appropriate multiple of the Haar measure on $G$, we may assume that $\operatorname{tr}(P)=1$. (If $G$ is discrete we may take $P$ to be the identity and thus keep the usual normalization for the Haar measure of $G$ ). Since $P$ contains no minimal projections, for each $\theta, 0<\theta<1$, there exists a subprojection $Q_{\theta} \subseteq P$ such that $\operatorname{tr}\left(Q_{\theta}\right)=\theta$. This is proved just as in the commutative case when instead of projections one deals with measurable sets containing no atom for a given measure (cfr. [2]). Using this fact we construct a sequence of partitions of $P$ as indicated in [2]. That is, we let $\pi_{n}=\left\{P_{n 1}, \cdots\right.$, $\left.P_{n 2^{n}}\right\}, P=P_{n 1}+\cdots+P_{n 2^{n}}, P_{n k} \in V N(G), \operatorname{tr}\left(P_{n k}\right)=1 / 2^{n}$. We also require that $\pi_{n+1}$ partitions each of the projections of $\pi_{n}$ into two projections of equal trace.

We define now the Rademacher operators $\left\{R_{n}\right\}_{n=1}^{\infty}$ and the Walsh operators $\left\{W_{n}\right\}_{n=0}^{\infty}$ as in [2], starting from the partitions $\left\{\pi_{n}\right\}$. Recall that $R_{n}=\sum_{k=1}^{2^{n}}(-1)^{k+1} P_{n k}$, where $\pi_{n}=\left\{P_{n 1}, \cdots, P_{n 2^{n}}\right\}$, and the projections of $\pi_{n}$ are indexed so that $P_{n-1, k}=P_{n, 2 k-1}+P_{n, 2 k}$ for $k=1, \cdots$, $2^{n-1}$. Recall also that $W_{0}=P$ and the Walsh operators $W_{n}$ are finite products of the Rademacher operators $R_{n}$, in such a way that $n=2^{j_{1}-1}+\cdots+2^{j_{s}-1}$, if $j_{1}<j_{2}<\cdots j_{s}$ and $W_{n}=R_{j_{1}} \cdots R_{j_{s}}$. The sequence $\left\{W_{n}\right\}_{n=0}^{\infty}$ is an orthonormal system in $L^{2}(\Gamma)$ which will be referred to as the Walsh system.

We define then the operators $\mathscr{E}_{n}$ mapping $L^{1}(\Gamma)$ into $L^{1}(\Gamma)$ as follows: 


$$
\mathscr{E}_{n}(T)=\sum_{k=0}^{2^{n}-1} \operatorname{tr}\left(W_{k} T\right) W_{k}, \quad \text { for } \quad T \in L^{1}(\Gamma) .
$$

We notice that if $\pi_{n}=\left\{P_{n 1}, \cdots, P_{n 2^{n}}\right\}$, then

$$
\mathscr{E}_{n}(T)=\sum_{j=1}^{2^{n}} m_{j_{n}}(T) P_{n j},
$$

where

$$
m_{j n}(T)=2^{n} \operatorname{tr}\left(P_{n j} T\right) .
$$

Therefore, since $\sum_{j=1}^{2^{n}}\left|\operatorname{tr}\left(P_{n j} T\right)\right| \leqq\|T\|_{L^{1}\left(\Gamma^{\prime}\right)}$, we also have

$$
\left\|\mathscr{E}_{n}(T)\right\|_{L^{1}(\Gamma)} \leqq\|T\|_{L^{1}(\Gamma)} \text {. }
$$

We shall define now an operator $\mathscr{E}$ of $L^{1}(\Gamma)$ into $L^{1}(\Gamma)$ which is the limit in the strong operator topology of the sequence $\mathscr{E}_{n}$. We start by defining $\mathscr{E}(V N)$ to be the commutative $v$. Neumann subalgebra of $V N$ generated by the projections $\left\{P_{n j}\right\}$. Since $\mathscr{E}(V N)$ is commutative, it is isomorphic to $L^{\infty}(Y, \mathscr{C}, m)$ where $Y$ is a set, $\mathscr{M}$ a $\sigma$-algebra of subsets of $Y$ and $m$ a positive measure of total mass one. ${ }^{1}$ Let $E_{n j}$ be the elements of $\mathscr{C}$ corresponding to $P_{n j}$. Then $m\left(E_{n j}\right)=\operatorname{tr}\left(P_{n j}\right)$ and the sets $\left\{E_{n j}\right\}$ generate $\mathscr{M}$. Now if $T \in$ $L^{1}(\Gamma)$ we can define a measure $\mu$ on $\mathscr{C}$ by letting $\mu\left(E_{n j}\right)=\operatorname{tr}\left(P_{n j} T\right)$ and $\mu(A)=\operatorname{tr}(Q T)$ where $A \in \mathscr{C}$ and $Q \in \mathscr{C}(V N)$ is the projection corresponding to $A$. The Radon-Nikodym derivative of $\mu$ with respect to $m$ is a function in $L^{1}(X, \mathscr{L}, m)$ which will be denoted by $\mathscr{E}(T)$. It is clear that $\mathscr{E}(T)$ can be identified with an element of $L^{1}(\Gamma)$. Furthermore $\mathscr{E}_{n}(\mathscr{E}(T))=\mathscr{E}\left(\mathscr{E}_{n}(T)\right)=\mathscr{E}_{n}(T)$. Since $\mathscr{E}(T)$ is a limit in $L^{1}(\Gamma)$ of linear combinations of the projections $P_{n j}$, we also have

$$
\left\|\mathscr{E}_{n}(T)-\mathscr{E}(T)\right\|_{L^{1}(\Gamma)} \longrightarrow 0 \text {. }
$$

3. The main theorem. We now have all the ingredients for the proof of our result.

THEOREM. There exists a positive definite function $f \in B(G)$ which is not in $A(G)$ and such that $\lim _{x \rightarrow \infty} f(x)=0$

Proof. Let $\left\{\alpha_{j}\right\}$ be a decreasing sequence of positive real numbers, bounded by one, and satisfying $\lim \alpha_{j}=0$ and $\sum \alpha_{j}^{2}=\infty$. Let $D$ be the Cantor group. For each $t=\left\{\varepsilon_{j}\right\} \in D$ we shall construct inductively a sequence $f_{n}^{t}$ of positive definite elements of $A(G)$, which converge uniformly to an element $f^{t} \in B_{\rho}$. We shall prove that for

$1 Y$ can be chosen to be $D$, the Cantor group with its Haar measure as shown in footnote 2). 
some $t \in D, f^{t} \notin A(G)$. We construct $f_{n}^{t}$ as the Fourier transform $\left(F_{n}^{t}\right)^{\wedge}(x)=\operatorname{tr}\left(L_{x}^{*} F_{n}^{t}\right)$ of operators $F_{n}^{t} \in \mathscr{E}(V N) \subseteq L^{1}(\Gamma)$. We refer to [11, p. 47] for properties of the transform $T^{\wedge}$ of an element $T$ of $L^{1}(\Gamma)$. We let $F_{1}=F_{1}^{t}=P$ for each $t \in D$. The operators $F_{n}^{t}$ will have the form

$$
F_{n}^{t}=\prod_{k=1}^{n-1}\left(P+r_{j_{k}}(t) \frac{\alpha_{k}}{2} R_{j_{k}}\right), \quad \text { for } \quad t=\left\{r_{j}(t)\right\} \in D,
$$

where the sequence $j_{k}$ will be determined inductively. Together with the sequence $F_{n}^{t}$ we shall construct a sequence of compact sets $K_{n}$, which will be used in the induction procedure. We let $K_{1}=$ $\left\{x \in G:\left|F_{1}^{\wedge}(x)\right| \geqq \alpha_{1} / 2\right\}$. Suppose that, for each $t, F^{t}, \cdots, F_{n}^{t}$ and $K_{1}, \cdots, K_{n-1}$, have been defined. We let $K_{n}=\left\{x \in G:\left|\left(F_{n}^{t}\right)^{\wedge}(x)\right| \geqq \alpha_{n} / 2\right.$, for some $t \in D\} \cup K_{n-1}$.

Notice that because of the form of $F_{n}^{t}$, as $t$ varies in $D$, we only have finitely many, in fact $2^{n-1}$, different operators $F_{n}^{t}$. Thus $K_{n}$ is the union of finitely many compact sets and it is compact. Let $\nu(x)=\sum_{j=0}^{\infty}\left|\operatorname{tr}\left(L_{x}^{*} W_{j}\right)\right|^{2}$. Then, as proved in [2], $\nu$ is a continuous function of $x$. Therefore, by Dini's theorem, for some positive integer $h_{n}$.

$$
\sum_{j \geq h_{n}}\left|\operatorname{tr}\left(L_{x}^{*} W_{j}\right)\right|^{2}<2^{-4 n}, \text { for } x \in K_{n} .
$$

Let $j_{n}$ be such that $j_{n}>j_{n-1}$, and $2^{j_{n}-1}>h_{n}$, and define

$$
F_{n+1}^{t}=F_{n}^{t}+r_{j_{n}}(t) \frac{\alpha_{n}}{2} R_{j_{n}} F_{n}^{t}, \quad \text { where } \quad t=\left\{r_{j}(t)\right\} \in D .
$$

We notice that $R_{j_{n}}=W_{2^{j_{n}}-1}$. Therefore all the Walsh operators appearing in the expansion of $R_{j_{n}} F_{n}^{t}$ have indices greater than $h_{n}$. As a consequence, if $W$ is any such Walsh operator,

$$
\left|\operatorname{tr}\left(L_{x}^{*} W\right)\right|<2^{-2 n}, \text { for } x \in K_{n} \text {. }
$$

Since the number of terms appearing in the expansion of $R_{j_{n}} F_{n}^{t}$ is exactly $2^{n-1}$, we deduce that

$$
\left|\operatorname{tr}\left(L_{x}^{*} R_{j_{n}} F_{n}^{t}\right)\right|<\left(\frac{1}{2}\right)^{n+1}, \quad \text { for } \quad x \in K_{n} .
$$

Now,

$$
\left(F_{n+1}^{t}\right)^{\wedge}(x)=\operatorname{tr}\left(L_{x}^{*} F_{n}^{t}\right)+r_{j_{n}}(t) \frac{\alpha_{n}}{2} \operatorname{tr}\left(L_{x}^{*} R_{j_{n}} F_{n}^{t}\right) .
$$

Therefore, for $x \notin K_{n}$,

$$
\left|\left(F_{n+1}^{t}\right)^{\wedge}(x)\right| \leqq \frac{\alpha_{n}}{2}+\frac{\alpha_{n}}{2}=\alpha_{n} .
$$


On the other hand, if $x \in K_{m} \backslash K_{m-1}$, with $m \leqq n$, then

$$
\left|\left(R_{j_{k}} F_{k}^{t}\right)^{\wedge}(x)\right|<\frac{1}{2^{k+1}}, \text { for } m \leqq k \leqq n .
$$

Therefore, if $x \in K_{m} \backslash K_{m-1}$,

$$
\begin{aligned}
&\left|\left(F_{n+1}^{t}\right)^{\wedge}(x)\right|= \mid \frac{\alpha_{n}}{2} r_{j_{n}}(t)\left(R_{j_{n}} F_{n}^{t}\right)^{\wedge}(x) \\
&+\cdots+\frac{\alpha_{m-1}}{2} r_{j_{m-1}}(t)\left(R_{j_{m-1}} F_{m-1}^{t}\right)^{\wedge}(x)+\left(F_{m-1}^{t}\right)^{\wedge}(x) \mid \\
& \leqq \\
& \frac{\alpha_{m-1}}{2}\left(\frac{1}{2^{n+1}}+\cdots+\frac{1}{2^{m}}\right)+\frac{\alpha_{m-1}}{2} \leqq \alpha_{m-1},
\end{aligned}
$$

(we have used the fact that the sequence $\alpha_{j}$ is decreasing). We have proved therefore that

$$
\left|\left(F_{n+1}^{t}\right)^{\wedge}(x)\right| \leqq \alpha_{j} \text { for } x \in K_{j}, j \leqq n .
$$

Since $K_{n+1} \supseteqq\left\{x:\left|\left(F_{n+1}^{t}\right)^{\wedge}(x)\right| \geqq \alpha_{n+1} / 2\right\}$, we can say that for all $n$ and $j \leqq n$,

$$
\left|f_{n}^{t}(x)\right|=\left|\left(F_{n}^{t}\right)^{\wedge}(x)\right| \leqq \alpha_{j} \quad \text { for } \quad x \notin K_{j} .
$$

Since for $t=\left\{r_{j}(t)\right\} \in D$,

$$
F_{n}^{t}=\prod_{k=1}^{n-1}\left(P+r_{j_{k}}(t) \frac{\alpha_{k}}{2} R_{j_{k}}\right),
$$

it follows that $F_{n}^{t}$ is a positive element of the commutative algebra $\mathscr{E}(V N)$ and $\operatorname{tr}\left(F_{n}^{t}\right)=\operatorname{tr}(P)=1$. We show now that $f_{n}^{t}$ converges uniformly (and hence weak ${ }^{*}$ ) to a positive definite function $f^{t} \in B_{\rho}$. Let $\varepsilon>0$ and $n$ be such that $2 \alpha_{n}<\varepsilon$. Then for all positive integers $p$,

$$
\left|f_{n}^{t}(x)-f_{n+p}^{t}(x)\right| \leqq 2 \alpha_{n}<\varepsilon \text { for } x \notin K_{n} .
$$

On the other hand if $x \in K_{n}$ and $i \geqq 0$, then $x \in K_{n+i}$, and hence

$$
\left|f_{n+i}^{t}(x)-f_{n+i+1}^{t}(x)\right|=\left|\frac{\alpha_{n+i}}{2}\left(R_{j_{n+\imath}} F_{n+i}^{t}\right)^{\wedge}(x)\right|<\frac{\alpha_{n+i}}{2} \frac{1}{2^{n+i}} .
$$

Thus for $x \in K_{n}$

$$
\left|f_{n}^{t}(x)-f_{n+p}^{t}(x)\right| \leqq \sum_{i=0}^{p-1}\left|f_{n+2}^{t}(x)-f_{n+\imath+1}^{t}(x)\right| \leqq \frac{\alpha_{n}}{2^{n}} .
$$

We conclude that $f_{n}^{t}$ is uniformly Cauchy and converges, uniformly and weak* to a positive definite function $f^{t} \in B_{\rho}$. Since $1=\operatorname{tr}\left(F_{n}^{t}\right)=$ $f_{n}^{t}(1)$ converges to $f^{t}(1)$, we have that $\left\|f^{t}\right\|_{B_{\rho}}=1$. 
It remains to prove that for at least some $t \in D, f^{t} \notin A(G)$. Suppose that for all $t, f^{t} \in A(G)$. We let $F^{t}$ be the element of $L^{1}(\Gamma)$ such that $\operatorname{tr}\left(L_{x}^{*} F^{t}\right)=f^{t}(z)$. We consider now an application $\Theta$ mapping $\mathscr{E}(V N)$ onto $L^{\infty}(D)$ and $\mathscr{E}\left(L^{1}\right)$ onto $L^{1}(D)$. We simply define $\Theta W_{n}=$ $w_{n}(t)$, where the $w_{n}(t)$ are the Walsh functions and extend $\Theta$ linearly and by continuity (weak* continuity for $V N$ ). ${ }^{2}$ We denote by $X$ the subspace of $L^{\infty}(D)$ which is the image under $\Theta$ of $\left\{\mathscr{E}(T): T \in C_{\rho}^{*}(G)\right\}$. Let $\beta_{j_{k}}=\alpha_{k} / 2$ and $\beta_{h}=0$ if $h=j_{k}$, and construct the functions $\phi_{n}$ as in the Lemma. Then

$$
\phi_{j_{n-1}}(s+t)=\prod_{k=1}^{n-1}\left(1+\frac{\alpha_{k}}{2} r_{j_{k}}(t) r_{j_{k}}(s)\right)=\left(\Theta F_{n}^{t}\right)(s),
$$

and $\phi_{k}=\phi_{j_{n}}$ for $j_{n} \leqq k<j_{n+1}$.

Let $T$ be an arbitrary element of $C_{\rho}^{*}(G)$, then

$$
\begin{aligned}
\operatorname{tr}\left(F^{t} T\right) & =\lim _{n} \operatorname{tr}\left(F_{n}^{t} T\right)=\lim _{n} \operatorname{tr}\left(F_{n}^{t} \mathscr{E}(T)\right) \\
& =\lim _{n} \int_{D} \phi_{n}(t+s) u(s) d s=\lim _{n} \phi_{n} * u(t),
\end{aligned}
$$

where $u \in X$ is the element $u=\Theta \mathscr{C}(T)$. On the other hand (cfr. the Lemma) the functions $\phi_{n}$ converge weak* (with respect to $C(D)$ ) to the measure $\mu$. Thus $\mu$ is a singular measure, because $\sum\left(\alpha_{k} / 2\right)^{2}=$ $+\infty$, and $\lim _{n} \mu\left(w_{n}\right)=0$. Since $\phi_{n}$ is a partial sum of the series of $\mu, \lim _{n}\left\|\hat{\phi}_{n}-\hat{\mu}\right\|_{\infty}=0$. This means that the operator on $L^{2}(D)$ of convolution by $\phi_{n}$ approaches in norm the operator of convolution by $\mu$. This implies that

$$
\lim _{n}\left\|\phi_{n} * u-\mu * u\right\|_{L^{2}(D)}=0
$$

It follows that

$$
\operatorname{tr}\left(F^{t} T\right)=\lim \phi_{n} * u(t)=\mu * u(t),
$$

the last equality holding for almost every $t \in D$. Since $G$ is separable the unit sphere of $V N$ is metrisable in the strong operator topology. ${ }^{3}$ Therefore given one of the Walsh operators $W$, there exists a sequence $T_{n}$ of elements of $C_{\rho}^{*}$, of norm less than or equal to one, which converges to $W$ in the strong operator topology. Let $u_{n}=$ $\Theta \mathscr{C}\left(T_{n}\right)$, then $u_{n}$ converges to $w$ in the weak* topology of $L^{\infty}(D)$,

${ }^{2}$ Consider the gage space $\Gamma=\left(L^{2}(G), V N(G), \operatorname{tr}\right)$ mentioned after the proof of the Lemma, and let $\mathscr{H}$ be the closed linear span of the Walsh operators $\left(W_{n}\right)_{n=0}^{\infty}$ in $L^{2}(G)$. Then the gage space $(\mathscr{H}, \mathscr{E}(V N)$, tr), where $\mathscr{E}(V N)$ acts on $\mathscr{H}$ by multiplication is unitarily equivalent to the commutative gage space $\left(L^{2}(D), L^{\infty}(D), m\right)$, where $D$ is the Cantor group and $m$ is its Haar measure. It follows from this that $\Theta$ has the asserted properties.

3 This is the only place where separability of $G$ is used. 
because $T_{n}$ converges to $W$ weak*, and $\mathscr{E}$ is weak* continuous from $V N$ to $\mathscr{E}(V N)$ (it is the adjoint of a continuous map between their respective pre-duals). For each $t \in D$

$$
\operatorname{tr}\left(F^{t} W\right)=\lim _{n} \operatorname{tr}\left(F^{t} T_{n}\right) .
$$

But $\operatorname{tr}\left(F^{t} T_{n}\right)=\mu * u_{n}(t)$ a.e., and convolution by a bounded measure is a weak* continuous map of $L^{\infty}(D)$ into $L^{\infty}(D)$. Thus $\lim _{n} \mu * u_{n}=$ $\mu * w$ in the weak* topology of $L^{\infty}$. On the other hand $\mu * u_{n}(t)$ converges pointwise to $\operatorname{tr}\left(F^{t} W\right)$, except for those $t$ which belong to the set $\left\{t \in D: \operatorname{tr}\left(F^{t} T_{n}\right) \neq \mu * u_{n}(t)\right.$ for some $\left.n\right\}$. Since this set is the countable union of sets of zero measure, $\mu * u_{n}(t) \rightarrow \operatorname{tr}\left(F^{t} T\right)$ a.e., and hence $\mu_{*} * u_{n} \rightarrow \operatorname{tr}\left(F^{t} T\right)$ in the weak* topology of $L^{\infty}$, from which it follows that $\operatorname{tr}\left(F^{t} W\right)=\mu * w(t)$, a.e., and, since the Walsh functions are denumerable,

$$
\operatorname{tr}\left(F^{t} W_{n}\right)=\mu * w_{n}(t)=\widehat{\mu}\left(w_{n}\right) w_{n}(t), \text { for all } n,
$$

except for a negligible set of $t \in D$. We fix $t \in D$ so that the above identity holds. It follows that $\operatorname{tr}\left(F^{t} W_{n}\right)=\hat{\phi}_{m}^{t}\left(w_{n}\right) w_{n}(t)$ if $n<2^{j_{n n}}$ and $\mathscr{E}_{j_{n}}\left(F^{t}\right)=F_{n}^{t}$. This means that $\lim _{n} F_{n}^{t}=\mathscr{E}\left(F^{t}\right)$ in $L^{1}(\Gamma)$, but if this were the case the functions $\phi_{n}^{t}$ would converge in $L^{1}(D)$ which is impossible because they are the partial sums of the FourierStieltjes series of the singular measure $\mu^{t}$, defined by $\mu^{t}(E)=\mu(E+t)$. We conclude that for at least one $t$ the function $f^{t}$ cannot be in $A(G)$ and the proof is complete.

Aknowledgement. In several earlier versions of this paper the proof that the positive definite function constructed was not in $A(G)$, was incomplete. We are grateful to the referee and/or to Bernard Russo for carefully detecting missing steps and hidden hypothesis.

\section{REFERENCES}

1. L. Bagget, A separable group having a discrete dual space is compact, J. Functional Anal., 10 (1972), 131-148.

2. C. Cecchini and A. Figà-Talamanca, Projections of uniqueness for $L^{p}(G)$, Pacific J. Math., 51 (1974), 37-47.

3. P. Eymard, L'algèbre de Fourier d'un groupe localement compact, Bull. Soc. Math. France, 92, (1964), 181-236.

4. A. Figà-Talamanca and G. I. Gaudry, Multipliers and sets of uniqueness of $L^{p}$, Michigan Math. J., 17 (1970), 179-191.

5. E. Hewitt and S. Zuckerman, Singular measures with absolutely continuous convolution squares, Proc. Camb. Phil. Soc., 62 (1966), 399.

6. I. Khalil, L'analyse harmonique de la droite et du groupe affine de la droite, Thèse de doctorat d'état, Université de Nancy, (1973).

7. A. Kleppner and R. Lipsman, The Plancherel formula for group extensions, Ann. Scient. de l'Ecole Norm. Sup., $4^{\circ}$ série t. 5 (1972), 459-516. 
8. R. A. Kunze, $L_{p}$ Fourier transforms on locally compact unimodular groups, Trans. Amer, Math. Soc., 89 (1958), 519-540.

9. G. Mauceri, Square integrable representations and the Fourier algebra of a unimodular group, (to appear).

10. M. D. Menchoff, Sur l'unicité du dévelopement trigonométrique, Comptes Rendus de l'Ac. de Sc. de Paris, 163 (1916), 433-436.

11. I. E. Segal, A noncommutative extension of abstract integration, Ann. of Math., 57 (1953), 401-457.

12. W. F. Stinespring, Integration theorems for gages and duality for unimodular groups, Trans. Amer. Math. Soc., 90 (1959), 15-56.

13. M. E. Walter, On a theorem of Figà-Talamanca, (to appear).

Received October 2, 1973 and in revised form October 13, 1976.

UNIVERSITY OF ROME

ROME, ITALY 



\section{PACIFIC JOURNAL OF MATHEMATICS}

\section{EDITORS}

RICHARD ARENS (Managing Editor)

University of California

Los Angeles, CA 90024

R. A. BEAUMONT

University of Washington

Seattle, WA 98105

C. C. Moore

University of California

Berkeley, CA 94720

\section{J. DugundJI}

Department of Mathematics

University of Southern California

Los Angeles, CA 90007

R. Finn and J. Milgram

Stanford University

Stanford, CA 94305

\section{ASSOCIATE EDITORS}

\section{E. F. BECKENBACH}

B. H. NEUMANN

F. WoLF

K. YOSHIDA

\section{SUPPORTING INSTITUTIONS}

\author{
UNIVERSITY OF BRITISH COLUMBIA \\ CALIFORNIA INSTITUTE OF TECHNOLOGY \\ UNIVERSITY OF CALIFORNIA \\ MONTANA STATE UNIVERSITY \\ UNIVERSITY OF NEVADA \\ NEW MEXICO STATE UNIVERSITY \\ OREGON STATE UNIVERSITY \\ UNIVERSITY OF OREGON \\ OSAKA UNIVERSITY
}

\author{
UNIVERSITY OF SOUTHERN CALIFORNIA \\ STANFORD UNIVERSITY \\ UNIVERSITY OF HAWAII \\ UNIVERSITY OF TOKYO \\ UNIVERSITY OF UTAH \\ WASHINGTON STATE UNIVERSITY \\ UNIVERSITY OF WASHINGTON \\ AMERICAN MATHEMATICAL SOCIETY
}

The Supporting Institutions listed above contribute to the cost of publication of this Journal, but they are not owners or publishers and have no responsibility for its content or policies.

Mathematical papers intended for publication in the Pacific Jaurnal of Mathematics should be in typed form or offset-reproduced, (not dittoed), double spaced with large margins. Please do not use built up fractions in the text of your manuscript. You may however, use them in the displayed equations. Underline Greek letters in red, German in green, and script in blue. The first paragraph or two must be capable of being used separately as a synopsis of the entire paper. Items of the bibliography should not be cited there unless absolutely necessary, in which case they must be identified by author and Journal, rather than by item number. Manuscripts, in triplicate, may be sent to any one of the editors. Please classify according to the scheme of Math. Reviews, Index to Vol. 39. All other communications should be addressed to the managing editor, or Elaine Barth, University of California, Los Angeles, California, 90024.

The Pacific Journal of Mathematics expects the author's institution to pay page charges, and reserves the right to delay publication for nonpayment of charges in case of financial emergency

100 reprints are provided free for each article, only if page charges have been substantially paid. Additional copies may be obtained at cost in multiples of 50 .

The Pacific Journal of Mathematics is issued monthly as of January 1966. Regular subscription rate: $\$ 7200$ a year (6 Vols., 12 issues). Special rate: $\$ 36.00$ a year to individual members of supporting institutions.

Subscriptions, orders for back numbers, and changes of address should be sent to Pacific Journal of Mathematics, 103 Highland Boulevard, Berkeley, California, 94708.

PUBLISHED BY PACIFIC JOURNAL OF MATHEMATICS, A NON-PROFIT CORPORATION

Printed at Kokusai Bunken Insatsusha (International Academic Printing Co., Ltd.). 8-8, 3-chome, Takadanobaba, Shinjuku-ku, Tokyo 160, Japan.

Copyrit (C) 1975 by Pacific Journal of Mathematics Manufactured and first issued in Japan 


\section{Pacific Journal of Mathematics \\ Vol. 69, No. $2 \quad$ June, 1977}

Carol Alf and Thomas Alfonso O'Connor, Unimodality of the Lévy spectral

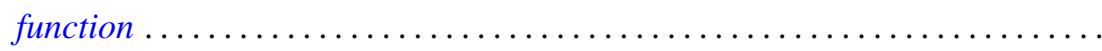

S. J. Bernau and Howard E. Lacey, Bicontractive projections and reordering of

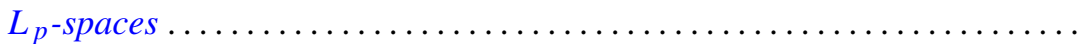

Andrew J. Berner, Products of compact spaces with bi-k and related spaces..... 303

Stephen Richard Bernfeld, The extendability and uniqueness of solutions of ordinary differential equations ...............................

Marilyn Breen, Decompositions for nonclosed planar m-convex sets ..........

Robert F. Brown, Cohomology of homomorphisms of Lie algebras and Lie

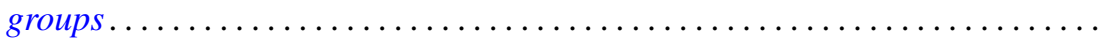

Jack Douglas Bryant and Thomas Francis McCabe, A note on Edelstein's iterative test and spaces of continuous functions ....................

Victor P. Camillo, Modules whose quotients have finite Goldie dimension ....... 333

David Downing and William A. Kirk, A generalization of Caristi's theorem with

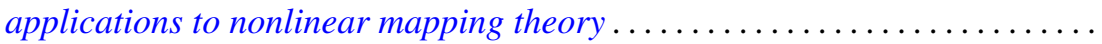

Daniel Reuven Farkas and Robert L. Snider, Noetherian fixed rings ...........

Alessandro Figà-Talamanca, Positive definite functions which vanish at

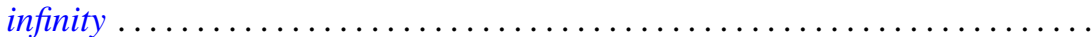

Josip Globevnik, The range of analytic extensions .................. 365

André Goldman, Mesures cylindriques, mesures vectorielles et questions de

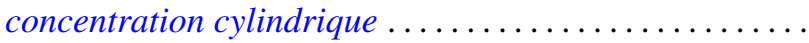

Richard Grassl, Multisectioned partitions of integers..........

Haruo Kitahara and Shinsuke Yorozu, A formula for the normal part of the

Laplace-Beltrami operator on the foliated manifold .... .

Marvin J. Kohn, Summability $R_{r}$ for double series .........

Charles Philip Lanski, Lie ideals and derivations in rings with involution ..

Solomon Leader, A topological characterization of Banach contractions . .

Daniel Francis Xavier O’Reilly, Cobordism classes of fiber bundles . .

James William Pendergrass, The Schur subgroup of the Brauer group . .

Howard Lewis Penn, Inner-outer factorization of functions whose Fourier series

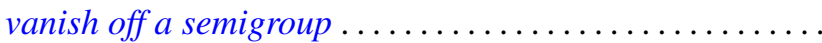

501

William T. Reid, Some results on the Floquet theory for disconjugate definite

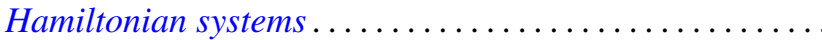

Caroll Vernon Riecke, Complementation in the lattice of convergence

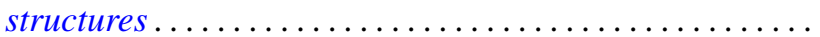

Louis Halle Rowen, Classes of rings torsion-free over their centers ......... 527

Manda Butchi Suryanarayana, A Sobolev space and a Darboux problem ....... 535

Charles Thomas Tucker, II, Riesz homomorphisms and positive linear maps.... 551

William W. Williams, Semigroups with identity on Peano continua ........... 557

Yukinobu Yajima, On spaces which have a closure-preserving cover by finite 